\title{
Granzyme B's roundhouse kick against bacteria
}

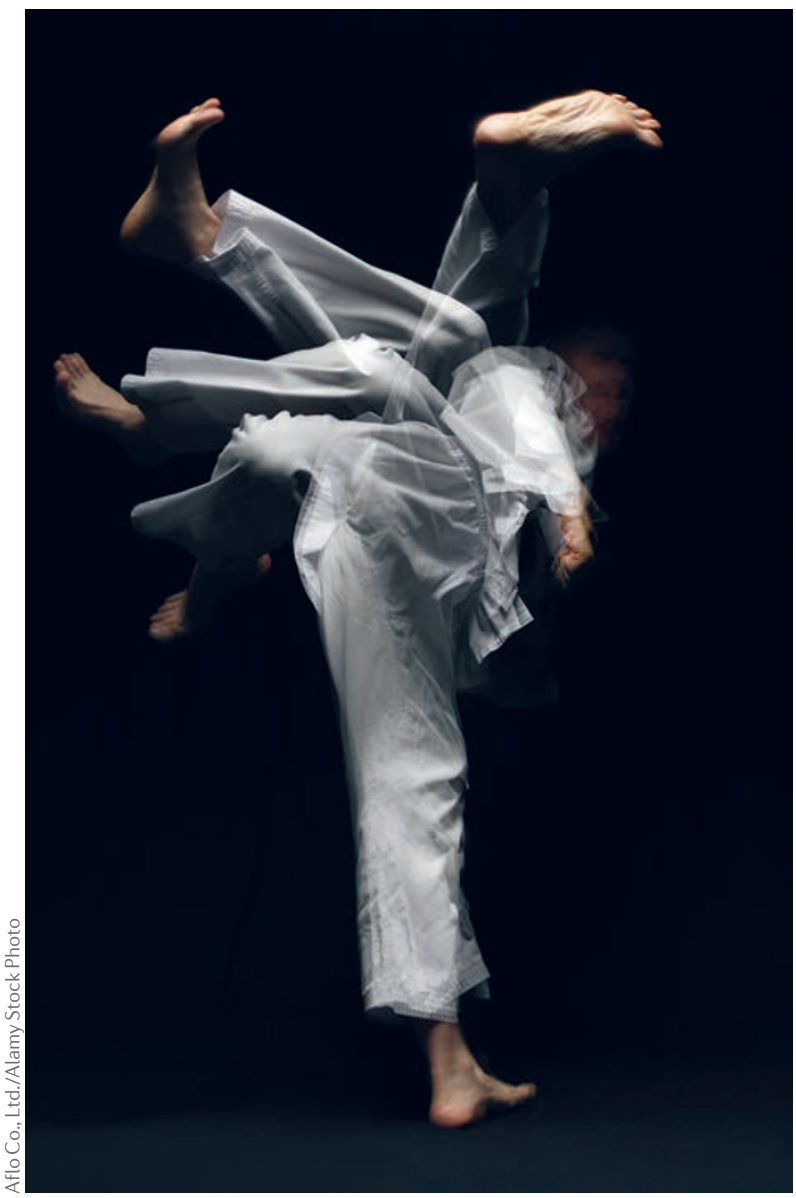

The protease granzyme $B$ is produced by natural killer cells and cytotoxic T lymphocytes and not only mediates the killing of infected cells but directly induces the death of intracellular bacteria. Previously, it was shown that granzyme B disrupts the electron transport chain and leads to oxidative damage and bacterial cell death; however, how bacteria that grow under anaerobic conditions are killed by the protease was unclear. Dotiwala et al. identify a new granzyme B-mediated cell death pathway species that targets central metabolism and protein synthesis in diverse bacteria and that does not require the presence of oxygen.

The authors identified potential bacterial targets of the protease using 2D isoelectric focusing (IEF)-SDSPAGE differential proteomics of cell lysates from Escherichia coli, Listeria monocytogenes and Mycobacterium tuberculosis that were incubated with active or proteolytically inactive granzyme B. Between $8 \%$ and $14 \%$ of bacterial proteins were targeted in the different species, and the targets mapped to several components of metabolic, biosynthetic and stress response pathways. Twenty targets occurred in all three bacterial species, including tRNA synthases, ribosome components, protein folding chaperones, Clp proteases and enzymes involved in central metabolism. This suggests that granzyme B kills bacteria by disrupting essential pathways that are conserved across bacterial species.

To confirm the inhibition of protein synthesis, the authors treated E. coli, L. monocytogenes and Mycobacterium smegmatis cultures with granzyme B in the presence of a reactive oxygen species scavenger, which inhibits the oxidative death pathway of the protease. Granzyme B inhibited protein synthesis, as measured by ${ }^{35} \mathrm{~S}$ incorporation, at similar levels to rifamycin and chloramphenicol, which are antibiotics that target transcription and translation, respectively. Furthermore, granzyme B cleaved ribosome components and are conserved across bacterial

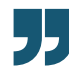

length in E. coli. Granzyme B also cleaved tRNA synthases in vitro and in vivo and inhibited their function, as measured by the production of pyrophosphate, a by-product of their ATPase function.

The proteomics data also indicated that protein quality control, in particular protein folding and the removal of misfolded proteins, is a target of granzyme B. Indeed, preincubation of the ribosome-associated chaperone trigger factor with the protease abolished its ability to refold guanidine hydrochloride-denatured GFP. Several members of the Clp machinery, which removes misfolded proteins, were also identified as granzyme B targets. The authors were able to show that ClpX, which was identified to be a target in all three bacteria that were tested and which unfolds misfolded proteins before they are degraded, lost its function in the presence of granzyme B. Granzyme B-pretreated ClpX was no longer able to unfold GFP that carried an ssrA tag, which is a marker of stalled translation and a signal for degradation.

In this series of experiments, Dotiwala et al. have shown that granzyme B disrupts several metabolic and biosynthethic pathways that are essential for the survival of bacterial cells. They call this process 'microptosis' for microbial programmed cell death, as it mirrors the proteolytic disruption of several important pathways during eukaryotic apoptosis. Notably, unlike antibiotics, which usually have one target and thus are prone to resistance development, granzyme B has a wide range of targets and so resistance due to target mutation is unlikely to occur. This multifunctionality also means that granzyme B can target diverse bacteria that live under very different environmental conditions.

Ursula Hofer

ORIGINAL ARTICLE Dotiwala, Fetal.

Granzyme B disrupts central metabolism and protein synthesis in bacteria to promote an immune cell death program. Cell http://doi. org/10.1016/j.cell.2017.10.004 (2017) 